\title{
ESTIMATIVA DA ÁREA FOLIAR DA MANGUEIRA ( Mangifera indica L.) cvs. TOMMY ATKINS E HADEN, UTILIZANDO DIMENSÕES LINEARES ${ }^{1}$
}

\author{
EUGÊNIO CELSO EMÉRITO ARAÚJO², ELIZANDRA PAULINO DOS SANTOS ${ }^{3}$, \\ CARLOS HENRIQUE BRITTO DE ASSIS PRADO ${ }^{4}$
}

\begin{abstract}
RESUMO - Os trabalhos existentes sobre a estimativa da área foliar da mangueira são raros e de resultados pouco convergentes. O objetivo deste trabalho foi determinar as equações que melhor descrevem a relação entre as dimensões lineares (comprimento e largura máxima) e a área da folha da mangueira, cultivares Tommy Atkins e Haden, possibilitando a estimativa rápida e não-destrutiva da área foliar (AF) utilizando apenas o comprimento (C), a largura máxima (L), ou ambos. A área foliar da mangueira pode ser estimada multiplicando-se o parto do comprimento pela largura, pelo fator 0,74 (para a cultivar Tommy Atkins) ou 0,78 (para a cultivar Haden), bem como pelas equações: $A F=4,96349 \mathrm{C}-33,42976\left(\mathrm{R}^{2}=0,86\right) ; \mathrm{AF}=17,02964 \mathrm{~L}$ $-18,88065\left(\mathrm{R}^{2}=0,85\right)$ e $A F=0,73499(\mathrm{CxL})+0,59459\left(\mathrm{R}^{2}=0,92\right)$ para a cultivar Tommy Atkins ou $\mathrm{AF}=5,35282 \mathrm{C}-33,17061\left(\mathrm{R}^{2}=0,88\right) ; \mathrm{AF}=19,09951 \mathrm{~L}-$ $24,61777\left(\mathrm{R}^{2}=0,89\right)$ e $A F=0,76015(\mathrm{CxL})+0,43257\left(\mathrm{R}^{2}=0,99\right)$ para a cultivar Haden.
\end{abstract}

Termos para indexação: área de folha, comprimento e largura de folhas, método não-destrutivo

\section{LEAF AREA ESTIMATION OF MANGO (Mangifera indica L.) cvs TOMMY ATKINS AND HADEN, USING LINEAR DIMENSIONS}

\begin{abstract}
Reports on leaf area estimation in mango is rare and with little convergent results. The objective of this work was to determine the equations that best describe the relationship among the linear dimensions (length and maximum width) and the leaf area (LA) of mango cvs Tommy Atkins and Haden, allowing the fast and non-destructive estimation of the leaf area just using the length (L), the maximum width (W), or both. The leaf area of mango can be obtained multiplying the product of the length for the width, and by the factor 0,74 (in Tommy Atkins cultivar) or by 0,78 (in Haden cultivar), as well as using the equations: $\mathrm{LA}=4,96349 \mathrm{~L}-33,429\left(\mathrm{R}^{2}=0,86\right) ; \mathrm{LA}=17,02964 \mathrm{~W}-18,88065\left(\mathrm{R}^{2}=0,85\right)$ and $\mathrm{LA}=0,73499$ $(\mathrm{LxW})+0,59459\left(\mathrm{R}^{2}=0,92\right)$ in Tommy Atkins cultivar or $\mathrm{LA}=5,35282 \mathrm{~L}-33,17061\left(\mathrm{R}^{2}=0,88\right) ; \mathrm{LA}=19,09951 \mathrm{~W}-24,61777\left(\mathrm{R}^{2}=0,89\right)$ and $\mathrm{LA}=0,76015(\mathrm{LxW})$ $+0,43257\left(\mathrm{R}^{2}=0,99\right)$ in Haden cultivar.
\end{abstract}

Index terms: leaf length and width, non-destructive method.

Os métodos para a obtenção da área foliar podem ser ou não destrutivos. Dentre os destrutivos estão: método planimétrico, método gravimétrico, utilização do peso seco da folha e de sua relação com a área foliar, dentre outros. Entre os métodos não-destrutivos destacamse: utilização da relação entre as medidas lineares da folha e sua área, método de contagem de quadrados preenchidos pelo contorno das folhas, planimetria fotoelétrica, planimetria fotográfica fotoelétrica, planimetria com radiação, fotografia hemisférica e outros (Kvet \& Marshall, 1971).

Os métodos destrutivos apresentam os incovenientes de não se aplicarem quando a quantidade de amostras é limitada, quando se deseja avaliar outras características além da área ao longo do tempo na mesma amostra e, geralmente, são grandes consumidores de tempo. Por outro lado, os métodos não-destrutivos poupam as amostras e, com a utilização de equipamentos modernos, são rápidos e precisos. Entretanto, em função do preço, esses equipamentos nem sempre são de fácil aquisição. Assim, a estimativa da área foliar utilizando a relação entre as dimensões lineares da folha e a respectiva área destaca-se como alternativa simples, barata e acessível, necessitando apenas de régua e cálculos associados. Nesse método, o procedimento mais utilizado consiste na determinação das dimensões lineares (comprimento e largura máxima) e da área real (por meio de algum método direto, como o gravimétrico, o planimétrico ou outro) da folha. O quociente entre a área real e o produto do comprimento pela largura máxima, é o termo denominado "fator de correção", o qual estima a área de qualquer outra folha da espécie, ao ser multiplicado pelo produto de suas dimensões lineares (comprimento e largura máxima) (Clements \& Goldsmith 1924; Darrow, 1932).

Os trabalhos existentes sobre a estimativa da área foliar da mangueira são raros e de resultados pouco convergentes. Prasada et al. (1994), em seus estudos sobre estimativas de área foliar determinou um fator de correção no valor de 0,640 para a mangueira. Tyagi \& Devi (1988) utilizou o valor de 0,857 , o qual é um fator geral aplicado a folhas elipsóides (Šesták et. al., 1971) não sendo específico para a mangueira. O objetivo deste trabalho foi determinar as equações que melhor descrevem a relação entre as dimensões lineares (comprimento e largura máxima) e a área da folha da mangueira, cultivares Tommy Atkins e Haden, possibilitanto a estimativa rápida e não-destrutiva da área foliar utilizando apenas o comprimento, a largura, ou ambos.

Foram utilizadas nove mudas de mangueira da cultivar Tommy Atkins e nove mudas da cultivar Haden. As mudas (com porta-enxerto da cultivar Espada) foram adquiridas no viveiro Manga Rosa (Bonfim Paulista, SP), em dezembro de 2003, quando contavam um ano de idade. Após a aquisição, foi feita uma poda de ramos e folhas deixando-se apenas o primeiro entrenó do enxerto. As mudas foram mantidas em estufa com 30\% de sombreamento, até março de 2004 quando todas as novas folhas emitidas já haviam atingido o crescimento máximo, sendo então retiradas (251 para Tommy e 122 para Haden) por meio de corte com tesoura de poda no ponto de união do pecíolo com a lâmina foliar. Foi determinado o comprimento e a largura máxima de cada folha, utilizando-se de uma régua de $30 \mathrm{~cm}$. Imediatamente, as folhas foram fotocopiadas. O contorno das folhas foi recortado do papel cuja massa foi determinada em balança analítica com precisão de mg (Mettler AE 260). A área foliar real foi então calculada por meio da relação com a massa do papel de área conhecida (método gravimétrico). Utilizando-se das medidas lineares tomadas anteriormente (comprimento e largura), determinou-se, a partir do produto, a área do retângulo que circunscreve a folha (área foliar calculada). O fator de correção foi então obtido pela divisão da área foliar real pela área foliar calculada (Clements \& Goldsmith, 1924; Darrow, 1932).

A área foliar foi também estimada por meio da regressão linear utilizando o comprimento, a largura e o produto do comprimento pela largura como variável independente e a área foliar real (calculada gravimetricamente) como variável dependente. O software Origin

5.0 (MicroCal Software, Inc., Northampton, MA, U.S.A) foi utilizado para as análises de regressão.

\footnotetext{
1 (Trabalho 034/2005). Recebido: 05/01/2005. Aceito para publicação: 09/08/2005.

${ }^{2}$ Eng $^{\circ}$. Agr ${ }^{\circ}$., MSc, Pesquisador Embrapa Meio-Norte, Doutorando PPGERN/UFSCAR, Caixa Postal 01, CEP 64006-220, Teresina-Piauí, emerito@cpamn.embrapa.br ${ }^{3}$ Graduanda Biologia, UFSCAR, São Carlos-São Paulo

${ }^{4}$ Prof. Dr., Departamento de Botânica, UFSCAR, São Carlos-SP.
} 
TABELA 1 - Valores médios (+ desvio-padrão-s), máximos e mínimos do comprimento (C), largura Máxima (L), área foliar real (Afr), área foliar calculada $(\mathrm{Afc}=\mathrm{CxL})$ e fator de correção $(\mathrm{f}=\mathrm{Afr} / \mathrm{Afc})$ para folhas de mangueira cvs Tommy Atkins e Haden. São Carlos (SP), março de 2004.

\begin{tabular}{ccccccc}
\hline & & $\mathrm{C}(\mathrm{cm})$ & $\mathrm{L}(\mathrm{cm})$ & $\mathrm{Afr}\left(\mathrm{cm}^{2}\right)$ & Afc $\left(\mathrm{cm}^{2}\right)$ & $\mathrm{f}$ \\
\hline \multirow{3}{*}{ Tommy Atkins } & Média $\pm \mathrm{s}$ & $13,23 \pm 3,53$ & $3,00 \pm 1,02$ & $32,25 \pm 18,84$ & $43,07 \pm 24,6$ & $0,74 \pm 0,07$ \\
& Máximo & 22,80 & 5,90 & 92,21 & 121,50 & 6,90 \\
& Mínimo & 6,10 & 1,00 & 5,42 & 0,48 & 0,40 \\
& Média $\pm \mathrm{s}$ & $13,64 \pm 4,92$ & $3,38 \pm 1,39$ & $39,83 \pm 28,05$ & $51,83 \pm 36,72$ & $0,78 \pm 0,12$ \\
& Máximo & 25,70 & 7,60 & 113,01 & 147,00 & 1,97 \\
& Mínimo & 4,70 & 1,10 & 5,29 & 6,58 & 0,30 \\
\hline
\end{tabular}

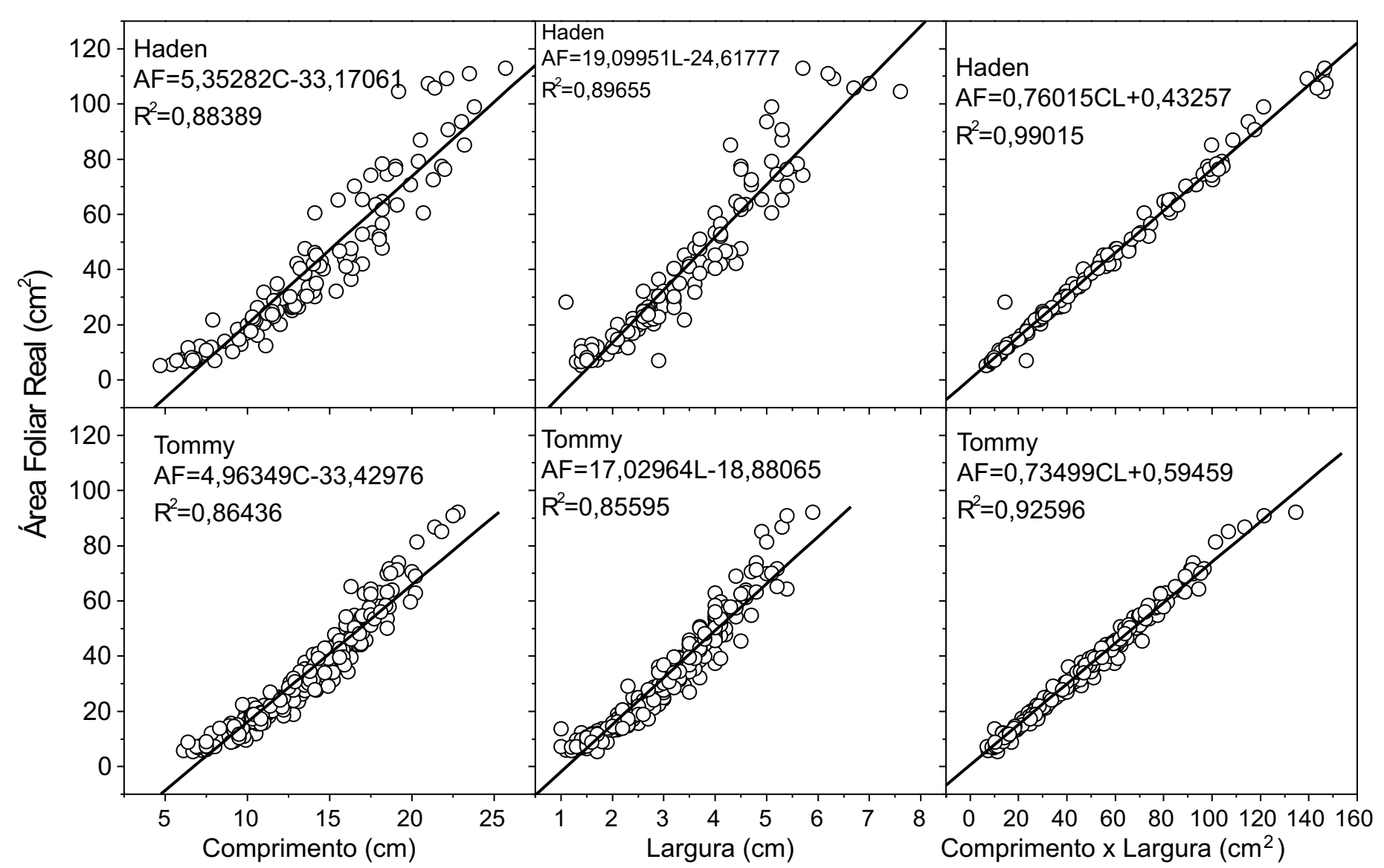

FIGURA 1 - Regressão linear entre a área foliar real (Afr) e o comprimento (C), a largura (L) e comprimento x largura (CxL) da mangueira cvs. Tommy Atkins e Haden. São Carlos (SP), março de 2004.

Os fatores de correção 0,74 e 0,78 (para as cultivares Tommy Atkins e Haden, respectivamente) obtidos neste trabalho (Tabela 1) diferem daqueles encontrados por Prasada (1994) e por Tyagi \& Devi (1988) $(0,640$ e 0,857 , respectivamente). Possivelmente, isto se deve às diferenças entre cultivares e condições de cultivo quanto ao trabalho de Prasada (1994) e ao uso de um fator geral para folhas elipsóides nãoespecífico para a mangueira, utilizado por Tyagi e Devi (1988).

As regressões lineares (Figura 1) demonstram que existe correlação estreita entre a área foliar e as dimensões lineares das folhas das duas cultivares. Isso implica que a área foliar dessas cultivares pode ser estimada utilizando-se das equações :

$\mathrm{AF}=4,96349 \mathrm{C}-33,42976\left(\mathrm{R}^{2}=0,86\right) \mathrm{I}$;

$\mathrm{AF}=17,02964 \mathrm{~L}-18,88065\left(\mathrm{R}^{2}=0,85\right) \mathrm{II}, \mathrm{e}$

$\mathrm{AF}=0,73499(\mathrm{CxL})+0,59459\left(\mathrm{R}^{2}=0,92\right) \mathrm{III}$

para a cultivar Tommy Atkins e:

$\mathrm{AF}=5,35282 \mathrm{C}-33,17061\left(\mathrm{R}^{2}=0,88\right) \mathrm{IV}$;

$\mathrm{AF}=19,09951 \mathrm{~L}-24,61777\left(\mathrm{R}^{2}=0,89\right) \mathrm{V}, \mathrm{e}$

$\mathrm{AF}=0,76015(\mathrm{CxL})+0,43257\left(\mathrm{R}^{2}=0,99\right) \mathrm{VI}$

para a cultivar Haden, onde :

$\mathrm{AF}=$ área foliar, $\mathrm{em}^{2}$;

$\mathrm{C}=$ comprimento, em $\mathrm{cm}, \mathrm{e}$

$\mathrm{L}=$ largura, em $\mathrm{cm}$

As estimativas que utilizam o produto do comprimento pela largura apresentam maior precisão (equações III e VI) que aquelas em que essas dimensões são usadas isoladamente (equações I, II, IV e V), como pode ser visto na Figura 1.

\section{REFERÊNCIAS}

CLEMENTS, F.E.; GOLDSMITH, G.W. The phytometer method in ecology. Whashington: Carnegie Inst. Whashington, 1924. 250p.

DARROW, G.M. Methods of measuring strawberry leaf areas. Plant physiology, Hanover, v. 7, p. 745-747, 1932.

KVET, J; MARSHALL, J.K. Assessment of leaf area and other assimilating plant surfaces. In: ŠESTÁK, Z.; ĖATSKY, J.; JARVIS, P.G. Plant photosynthetic production: manual of methods. Netherlands: Dr. W. Junk N.V. Publ. , 1971. p. 517-555.

PRASADA, R.G.S.L.H.V.; SIBY, S.; RAO, G. L. S. H. V. P.; SEBASTIAN, S. Estimation of leaf area in tree crops. Journal of Plantation Crops, Kasaragod, v. 22, n. 1, p. 44-46,1994.

ŠESTÁK, Z.; ĖATSKY, J.; JARVIS, P.G. Plant photosynthetic production: manual of methods. Netherlands: Dr. W. Junk N.V. Publ., 1971. $818 \mathrm{p}$.

TYAGI, D.N.; DEVI, K.M.T.M. Physiology of mango (Mangifera indica $L$.): I. Preliminary studies on the physiological characteristics of leaf. Indian Journal of Plant Physiology, New Delhi, v.31, n.4, p.368373, 1988. 